\title{
Rehabilitation as an Essential Element in the Clinical Practice of Orthopaedics and Traumatology
}

\section{Ivet B Koleva ${ }^{1,2 *}$ and Borislav Yoshinov ${ }^{3}$}

${ }^{1}$ Medical University of Sofia, Bulgaria

${ }^{2}$ Specialized Rehabilitation Hospital "Serdika", Sofia, Bulgaria

${ }^{3}$ Medical Faculty of Sofia University, Bulgaria

*Corresponding Author: Ivet B Koleva, Professor, Medical University of Sofia,

Bulgaria.
Received: August 24, 2020

Published: August 31, 2020

(C) All rights are reserved by Ivet B Koleva

and Borislav Yoshinov., et al.

\begin{abstract}
The World Report on Disability defines the goals of rehabilitation: prevention of the loss of function; slowing the rate of loss of function; improvement or restoration of function; compensation for lost function; maintenance of current function. Orthopedic rehabilitation is part of the rehabilitation sciences, needed frequently in clinical practice. Our opinion is that rehabilitation is an essential element in the patients' management of Orthopedics and Traumatology.

In this article, we present basic theoretical knowledge, necessary for a successful orthopedic rehabilitation, and we cite practical skills of rehabilitation in patients with the most frequent orthopedic conditions, including after orthopedic surgery. We explain our own proposal for OT rehabilitation algorithms, with the goal to stimulate the functionality of our patients and to assure their independence in activities of daily living, respectively their autonomy.
\end{abstract}

Keywords: Rehabilitation; Orthopedics and Traumatology; Physical Medicine; Electric Currents; Magnetic Field; Deep Oscillation; Cryotherapy

\section{Abbreviations}

ADL: Activities of Daily Living; ET: Ergotherapy; ICD: International Classification of Diseases; ICF: International Classification of Functioning; MMT: Manual Muscle Test; OT: Orthopedics and Traumatology; PRM: Physical and Rehabilitation Medicine; PT: Physiotherapy; QoL: Quality of Life; Rehab: Rehabilitation; UEMS: European Union of Medical Specialists

Rehabilitation is a functional therapy, realized in acute and chronic stage departments of hospitals for active or for long-term care; by a multi-disciplinary multi-professional team (medical doctors, nurses, physiotherapists, occupational therapists). The World Health Organization's (WHO) definition of rehabilitation is: "The use of all means aimed to reduce the impact of disabling and handicapping conditions, and at enabling people with disabilities to achieve optimal social integration". The World Report on Disability [1] of the World Health Organization and World Bank defines the goals of rehabilitation: prevention and slowing the rate of loss of function; improvement, restoration or compensation of lost function; maintenance of current function.

Orthopedic rehabilitation is part of the rehabilitation sciences, needed frequently in clinical practice.

According the Definition [2] amended by the Council of the European Union of Medical Specialists (UEMS): "The medical act encompasses all the professional action, e.g. scientific, teaching, training and educational, clinical and medico-technical steps, performed to promote health and functioning, prevent diseases, provide diagnostic or therapeutic and rehabilitative care to patients, individuals, groups or communities in the framework of the respect 
of ethical and deontological value. It is the responsibility of and must always be performed by a registered medical doctor/physician or under his or her direct supervision and/or prescription".

According the definitions [3-5] in the White Book of the specialty Physical and Rehabilitation Medicine (PRM), formulated by the UEMS - PRM Section and Board: PRM is an "independent medical specialty, oriented to the promotion of physical and cognitive functioning, activities (including environment), participation (including quality of life) and changes in personal factors and environment. It is thus responsible for the prevention, diagnosis, treatments and rehabilitation management of people with disabling medical conditions and co-morbidity across all ages".

PRM is a "Medicine of Functioning", focusing on the improvement of functioning. Our work is based on the International Classification of Diseases [ICD] [6] and on the International Classification of Functioning, disability and Health [ICF] [7].

The diagnosis in PRM is the interaction between the medical diagnosis and a PRM-specific functional assessment. Outcomes of PRM interventions and programs, showed reduction of impairments in body functions, activity limitations, impacting on participation restrictions, and also on reduction in costs as well as decrease in mortality for certain groups of patients.

The goal of PRM is prevention, treatment and rehabilitation. In the management of orthopedic patients, the role of rehabilitation is very important.

Interventions in PRM are either provided directly by PRM physicians or within the rehabilitation team, including medical doctors (specialists in Orthopedics and Traumatology, and in Physical and Rehabilitation Medicine); health professionals (nurses, physiotherapists, occupational therapists, etc).

Rehabilitation algorithm includes detailed functional evaluation (based on ICD and ICF) and synergic combination of physical modalities (movement, activities, mineral waters, electric currents, etc). The rehabilitation battery includes many natural physical modalities [water (mineral baths), air, sun, exercises, massage, manual therapy techniques (traction, mobilization, and manipulation); ergotherapy (work and activities)] and pre-formed physical modalities [electric currents, light, magnetic field, ultrasound, laser, etc].
PRM-interventions in the field of orthopedics and traumatology comprise a wide range of treatments, including medicines, physical therapies, exercises, education and many others. The principle of the rehabilitation 'puzzle' is respected - a synergic combination of physiotherapy, occupational therapy, cryo- or hydro-therapy, preformed modalities. Ultimately, our attention is oriented to some effective clinical and instrumental treatment methods [8]: functional electrical stimulations (with low and middle frequency electric currents); deep oscillation; manual therapy techniques (tractions, mobilizations and manipulations); proprioceptive neuro-muscular facilitation (PNF) methods; analytic exercises, device-assisted mechano-therapy (passive, active or combined), etc. We insist on the importance of technical aids (wheelchair, canes, or walking sticks) and weight bearing (restricted, fractional or total) during the rehabilitation process. Future possibilities are rising, as Michelangelo hand and C-leg (computerized leg in amputees); robotic-assisted rehabilitation with Exoskeleton and Locomat (for gait training), virtual reality rehabilitation (for grasp and gait training).

In our own modest clinical practice (of 30+years) we apply OT rehabilitation algorithms [8], with the goal to stimulate the functionality of our patients and to assure their independence in activities of daily living, respectively their autonomy. We use an integrative and holistic approach to the patient, based on ICF and on clinical principles. The OT-rehabilitation process according to the so-called "rehabilitation cycle" includes an assessment and definition of the (individual) rehabilitation goals, assignment to the rehabilitation program evaluation of individual outcomes. The general OT-rehabilitation therapeutic protocol includes one or two preformed modalities, one cryo-agent, two or three physiotherapeutic procedures (including analytic exercises, post-isometric relaxation, stretching techniques, massage, etc.).

We accentuate on grasp, balance and gait training, and on occupational therapy.

We are oriented to typical and atypical clinical cases, including patients with comorbidities, complex or multiple fractures, common or rare complications.

In patients with upper limb orthopedic problems and after orthopedic surgery, we emphasize on grasp and grip training and recovery of the functionality of the upper extremity limb in activities and self-service; e.g. after shoulder replacement, after distal radius fracture and Zudeck's algoneurodystrophy; etc. 
We pay special attention to rehabilitation after lower limb orthopedic surgery: in patients with acetabular, inter/trans trochanteric femoral fractures, with gamma nail or screw - plaque metallic osteosynthesis; joint replacement of lower extremities (hip and knee arthroplasty); anterior cruciate ligament (ACL) alloplasty; meniscectomy. We have rehabilitation protocols for patients with phantom pain, after trans-femoral and trans-tibial amputations.

In conclusion, we must underline that orthopedic rehabilitation is an essential element in the clinical practice with serious impact on patients' functionality, autonomy and quality of life.

\section{Bibliography}

1. World Health Organization. "World Report on Disability" (2012).

2. UEMS (Union Européenne des Médecins Spécialistes) 2005/14 final. European Definition of the Medical Act. Adopted in Munich (2005).

3. UEMS - PRM-section: Definition of Physical and Rehabilitation Medicine (2005).

4. C Gutenbrunner., et al. "White Book on Physical and Rehabilitation Medicine in Europe. Produced by the Section of Physical and Rehabilitation Medicine, Union Européenne des Médecins Spécialistes (UEMS), the European Board of Physical and Rehabilitation Medicine and l'Académie Européenne de Medicine de Réadaptation in conjunction with the European Society of Physical and Rehabilitation Medicine (ESPRM)". Journal of Rehabilitation Medicine 45.1 (2007): 1-48.

5. White Book on Physical and Rehabilitation Medicine (PRM) in Europe. Chapter 7. The clinical field of competence: PRM in practice. European Physical and Rehabilitation Medicine Bodies Alliance. European Journal of Physical and Rehabilitation Medicine 54.2 (2018): 230-260.

6. World Health Organization. The International Statistical Classification of Diseases and Health-Related problems ICD-10. Second Edition. Geneva: WHO (2005).

7. World Health Organization. International Classification of Functioning, Disability and Health (ICF). WHO, Geneva (2001).

8. Koleva I. Orthopedic rehabilitation. E-book. Sofia: SIMEL (2020): 498.

\section{Assets from publication with us}

- Prompt Acknowledgement after receiving the article

- Thorough Double blinded peer review

- Rapid Publication

- Issue of Publication Certificate

- High visibility of your Published work

Website: https://www.actascientific.com/

Submit Article: https://www.actascientific.com/submission.php

Email us: editor@actascientific.com

Contact us: +919182824667 